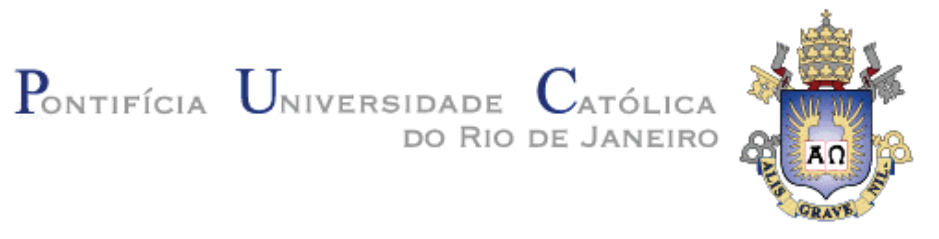

Thiago Delgado Pinto

\title{
Uma Ferramenta para Geração e Execução Automática de Testes Funcionais Baseados na Descrição Textual de Casos de Uso
}

Dissertação de Mestrado

Dissertação apresentada como requisito parcial para a obtenção do grau de Mestre pelo Programa de Pós-graduação em Informática do Departamento de Informática do Centro Técnico Científico da PUC-Rio.

Orientador: Prof. Arndt von Staa

Rio de Janeiro Abril de 2013. 


\section{Pontifícia $U_{\text {niversidade }}$ Católica $_{\text {a }}$ \\ Thiago Delgado Pinto}

\section{Uma Ferramenta para Geração e Execução Automática de Testes Funcionais Baseados na Descrição Textual de Casos de Uso}

Dissertação apresentada como requisito parcial para a obtenção do grau de Mestre pelo Programa de Pós-graduação em Informática do Departamento de Informática do Centro Técnico Científico da PUC-Rio. Aprovada pela Comissão Examinadora abaixo assinada.

Prof. Arndt von Staa

Orientador e Presidente

Departamento de informática - PUC-Rio

Prof. ${ }^{a}$ Simone Diniz Junqueira Barbosa

Departamento de informática - PUC-Rio

Prof. Alessandro Fabrício Garcia Departamento de informática - PUC-Rio

Prof. José Eugenio Leal

Coordenador Setorial do Centro

Técnico Científico - PUC-Rio

Rio de Janeiro, 05 de Abril de 2013. 
Todos os direitos reservados. É proibida a reprodução total ou parcial do trabalho sem autorização da universidade, do autor e do orientador.

\section{Thiago Delgado Pinto}

É bacharel em Informática e especialista em Engenharia de Software. Trabalhou por mais de 10 anos com desenvolvimento de software, atuando como analista/desenvolvedor, arquiteto de software e líder técnico. É professor do CEFET/RJ, onde leciona disciplinas relacionadas à Engenharia de Software, Desenvolvimento de Software e Sistemas Operacionais.

Ficha Catalográfica

Pinto, Thiago Delgado

Uma ferramenta para geração e execução automática de testes funcionais baseados na descrição textual de casos de uso / Thiago Delgado Pinto; Orientador: Arndt von Staa. - 2013.

190 f.: il. ; $30 \mathrm{~cm}$

Dissertação (mestrado) - Pontifícia Universidade Católica do Rio de Janeiro, Departamento de Informática, 2013.

Inclui bibliografia

1. Informática - Testes. 2. Engenharia de Software. 3. Caso de uso. 4. Teste funcional. 5. Geração de casos de teste. 6. Geração de valores. 7. Geração de código de teste. 8. Automação da execução do teste. I. Staa, Arndt von. II. Pontifícia Universidade Católica do Rio de Janeiro. Departamento de Informática. III. Título. 
Para Idaiane, pela companhia em todos os momentos. 


\section{Agradecimentos}

À minha esposa, Idaiane, pelo apoio, carinho e compreensão, que me deram forças nos momentos mais difíceis.

Ao professor e orientador Arndt, pelas prazerosas conversas, valiosas reflexões e importantes direcionamentos.

Aos membros da banca, pelas observações que possibilitaram melhorar este trabalho.

À PUC-Rio e ao CEFET/RJ, que possibilitaram a realização do mestrado.

Aos meus familiares e amigos, por entenderem as ausências. 


\section{Resumo}

Pinto, Thiago Delgado; Staa, Arndt von (Orientador). Uma Ferramenta para Geração e Execução Automática de Testes Funcionais Baseados na Descrição Textual de Casos de Uso. Rio de Janeiro, 2013. 190p. Dissertação de Mestrado - Departamento de Informática, Pontifícia Universidade Católica do Rio de Janeiro.

Esta dissertação apresenta uma solução para a geração e execução automática de testes funcionais a partir da descrição textual de casos de uso, visando verificar se determinada aplicação atende aos requisitos funcionais definidos por esta documentação. A ferramenta construída é capaz de gerar casos de teste semânticos valorados, transformá-los em código-fonte (para Java Swing e os frameworks de teste TestNG e FEST, na versão atual), executá-los, coletar os resultados e analisar se os casos de uso da aplicação atendem ou não a estes requisitos. Dentre os principais diferenciais da solução construída estão a cobertura de cenários de teste que envolvem múltiplos casos de uso, a cobertura de cenários envolvendo recursão, a possibilidade da definição de regras de negócio sobre dados existentes em bancos de dados de teste, a geração automática dos valores fornecidos nos testes e a geração de testes funcionais semânticos, num formato independente de linguagem de programação e frameworks de teste.

\section{Palavras-chave}

Engenharia de software; Caso de uso; Teste funcional; Geração de casos de teste; Geração de valores; Geração de código de teste; Automação da execução do teste. 


\section{Abstract}

Pinto, Thiago Delgado; Staa, Arndt von (Advisor). A tool for the automatic generation and execution of functional tests based on the textual use case description. Rio de Janeiro, 2013. 190p. MSc Dissertation - Departamento de Informática, Pontifícia Universidade Católica do Rio de Janeiro.

This master's dissertation presents a solution for the automatic generation and execution of functional tests based on the textual use case description and aims to verify whether certain application matches its functional requirements defined by this documentation. The constructed tool is capable of generating valued semantic test cases, of transforming them into source code (for Java Swing and the TestNG and FEST frameworks, in the current version), of executing them, of collecting the results and of analyzing whether the application's use cases matches (or not) its requirements. The solution main differentials includes the coverage of test scenarios that involves more than one use case, the coverage of scenarios containing recursive flows, the possibility of defining business rules using data existing in test databases, as well as the automatic generation of test values, and the generation of semantic functional tests in a format independent of programming languages and frameworks.

\section{Keywords}

Software engineering; Use case, Functional testing; Test case generation; Value generation; Test code generation; Test execution automation. 


\section{Sumário}

1. Introdução 15

$\begin{array}{ll}\text { 1.1. Objetivos } & 16\end{array}$

$\begin{array}{ll}\text { 1.2. Resultados esperados } & 17\end{array}$

1.3. Organização dos capítulos seguintes 18

2. Terminologia 19

2.1. Descrição textual de casos de uso 19

2.2. Decomposição de casos de uso 21

2.3. Descrição de fluxos de um caso de uso 21

2.4. Geração de cenários 22

2.5. Combinação de cenários 23

2.6. Geração de casos de teste 23

2.7. Máquina de estados generalizada 24

2.8. Forma de Backus-Naur (BNF) 24

3. Estado da Arte 26

3.1. Trabalhos parcialmente correlatos 26

3.2. Principais trabalhos correlatos 36

3.3. Comparativo 51

4. Metodologia 54

4.1. Visão geral

4.2. Descrição textual de casos de uso (Etapa 1) 56

4.3. Detalhamento das regras de negócio (Etapa 2) 62

4.4. Geração de cenários para cada caso de uso (Etapa 3) 67

4.5. Combinação de cenários entre casos de uso (Etapa 4) 73

4.6. Geração de casos de teste semânticos (Etapa 5) 77

4.7. Transformação em código-fonte (Etapa 6) 86

4.8. Execução do código-fonte (Etapa 7) 87 
4.9. Coleta, pré-análise e transformação dos resultados de execução (Etapa 8)

4.10. Coleta, análise e apresentação dos resultados (Etapa 9) 90

5. Aspectos de Projeto e Implementação 91

$\begin{array}{ll}\text { 5.1. Aspectos de qualidade } & 91\end{array}$

5.2. Ferramentas e tecnologias 94

$\begin{array}{ll}\text { 5.3. Arquitetura } & 99\end{array}$

6. Prova de Conceito 131

6.1. Seleção do software 131

6.2. Adaptação do software 131

6.3. Seleção dos casos de uso 132

6.4. Especificação dos requisitos 133

6.5. Considerações sobre as especificações 134

6.6. Considerações sobre os testes 135

6.7. Especificação baseada no SST 135

6.8. Versão levemente divergente da especificação 156

6.9. Versões do SST com mutantes 158

7. Conclusões 169

7.1. Contribuições do trabalho 170

$\begin{array}{ll}\text { 7.2. Resultados alcançados } & 171\end{array}$

8. Projetos Futuros 173

8.1. Melhorias 173

8.2. Avaliações 175

8.3. Funcionalidades complementares 175

$\begin{array}{ll}\text { Referências Bibliográficas } & 177\end{array}$

$\begin{array}{lr}\text { Apêndice A } & 182\end{array}$

$\begin{array}{lr}\text { Anexo A } & 187\end{array}$ 


\section{Lista de Figuras}

Figura 1 - Processo realizado pela ferramenta construída ........................54

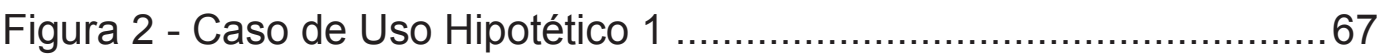

Figura 3 - Caso de Uso Hipotético 2 ……....................................... 70

Figura 4 - Dependências de pacotes Java …………...........................95

Figura 5 - Componentes envolvidos na solução..................................... 101

Figura 6 - Classes no contexto de um software …………..................106

Figura 7 - Classes no contexto dos fluxos de um caso de uso...............108

Figura 8 - Classes no contexto de um passo ……………………......109

Figura 9 - Classes no contexto de elementos de um caso de uso .........111

Figura 10 - Classes relacionadas a um Perfil .....................................112

Figura 11 - Classes no contexto de uma Regra de Negócio ..................114

Figura 12 - Classes no contexto dos CTSVO .....................................116

Figura 13 - Classes no contexto do resultado da execução dos testes.. 118

Figura 14 - Classes relacionadas à geração de valores..........................122

Figura 15 - Classes relacionadas à geração de valores para um

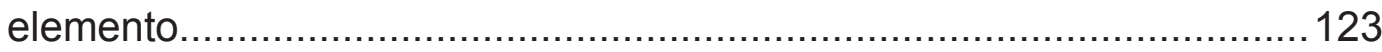

Figura 16 - Classes relacionadas à geração de uma suíte de testes .....125

Figura 17 - Classes relacionadas à geração de métodos de teste semânticos com valores inválidos

Figura 18 - Classes relacionadas à geração de métodos de teste semânticos com valores válidos 128

Figura 19 - Classes que representam a estrutura do relatório gerado

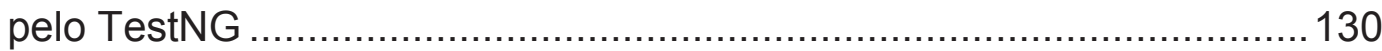

Figura 20 - Tela com os resultados da execução dos testes .................150

Figura 21 - Primeiro exemplo de detalhes sobre falha ......................... 151

Figura 22 - Segundo exemplo de detalhes sobre falha ........................153 


\section{Lista de Tabelas}

Tabela 1 - Formulário exemplo para a descrição textual de casos de

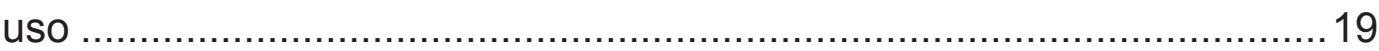

Tabela 2 - Exemplo de fluxos de um caso de uso ..................................21

Tabela 3 - Significado de algumas expressões usadas neste trabalho ....25

Tabela 4 - Enfoque de trabalhos parcialmente correlatos .......................27

Tabela 5 - Operadores mutantes para casos de uso ............................42

Tabela 6 - Panorama das principais ferramentas encontradas .................48

Tabela 7 - Campos usados na descrição textual de casos de uso............56

Tabela 8 - Fontes de valores para Regras de Negócio ............................65

Tabela 9 - Tipos de caso de teste elaborados........................................ 79

Tabela 10 - Principais ferramentas utilizadas .........................................94

Tabela 11 - Principais plug-ins para Eclipse utilizados............................95

Tabela 12 - Principais tipos de dados de domínio do modelo

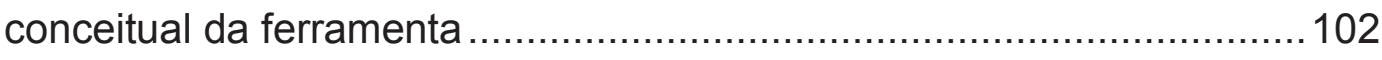

Tabela 13 - Principais tipos de dados relacionados ao

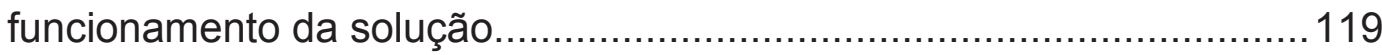

Tabela 14 - Descrição textual do caso de uso Efetuar Login .................136

Tabela 15 - Descrição textual do caso de uso Acessar Sistema............137

Tabela 16 - Descrição textual do caso de uso Cadastrar Cliente ...........137

Tabela 17 - Descrição textual do caso de uso Adicionar Animal............140

Tabela 18 - Descrição textual do caso de uso Remover Animal .............141

Tabela 19 - Detalhes dos arquivos de código gerados ..........................143

Tabela 20 - Desempenho da execução dos testes ...............................147

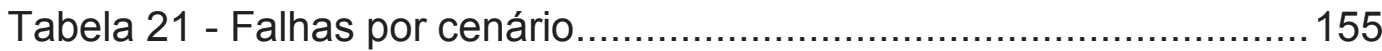

Tabela 22 - Tempos envolvidos na execução dos testes para a versão com especificação levemente divergente ................................157

Tabela 23 - Falhas por cenário na versão levemente divergente........... 158

Tabela 24 - Mutações realizadas por caso de uso, para o primeiro

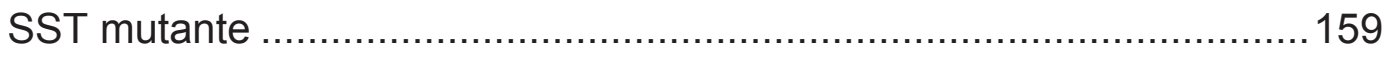

Tabela 25 - Distribuição das falhas por cenário usando Adicionar 
Animal com o primeiro mutante

Tabela 26 - Tempos de execução para os testes usando Adicionar Animal com o primeiro mutante.

Tabela 27 - Distribuição das falhas por cenário usando Cadastrar Cliente com o primeiro mutante.

Tabela 28 - Tempos de execução para os testes usando Cadastrar Cliente com o primeiro mutante

Tabela 29 - Distribuição das falhas por cenário usando Efetuar Login com o primeiro mutante

Tabela 30 - Tempos de execução para os testes usando Efetuar Login com o primeiro mutante

Tabela 31 - Resultados consolidados para o primeiro mutante.

Tabela 32 - Mutações realizadas por caso de uso, para o segundo SST mutante

Tabela 33 - Distribuição das falhas por cenário usando Adicionar Animal com o segundo mutante

Tabela 34 - Tempo de execução para os testes usando Adicionar Animal com o segundo mutante

Tabela 35 - Distribuição das falhas por cenário usando Cadastrar Cliente com o segundo mutante

Tabela 36 - Tempos de execução para os testes usando Cadastrar Cliente com o segundo mutante

Tabela 37 - Distribuição das falhas por cenário usando Efetuar Login com o segundo mutante

Tabela 38 - Tempos de execução para os testes usando Efetuar Login com o segundo mutante

Tabela 39 - Resultado consolidado de execução para o segundo mutante 


\section{Listagens}

Listagem 1 - Sintaxe de um passo de um fluxo .................................61 61

Listagem 2 - Algoritmo de geração de cenários de um caso de uso ........72

Listagem 3 - Algoritmo em pseudocódigo da combinação de cenários....76

Listagem 4 - Sintaxe dos casos de teste semânticos valorados ..............82

Listagem 5 - Algoritmo em pseudocódigo para geração de método de teste cujos elementos contêm valores válidos

Listagem 6 - Algoritmo em pseudocódigo para geração de método

de teste que verifica elementos obrigatórios

Listagem 7 - Algoritmo em pseudocódigo para conversão de testes

semânticos valorados em código-fonte

Listagem 8 - Sintaxe dos resultados da execução dos testes

Listagem 9 - Exemplo de teste de integração realizado no projeto

Listagem 10 - Exemplo de teste parametrizável criado com TestNG

Listagem 11 - Exemplo de teste com número de execuções

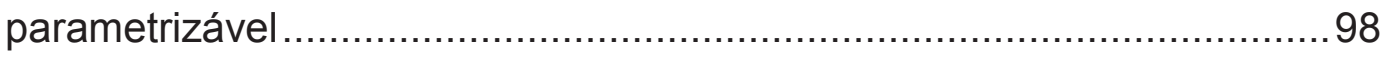

Listagem 12 - Exemplo de código que usa o framework FEST Swing .....99

Listagem 13 - Correção do método Main no SST ...............................132

Listagem 14 - Trecho de arquivo JSON com CTSVO ..........................143

Listagem 15 - Exemplo de código-fonte de teste gerado pela ferramenta

Listagem 16 - Trecho de arquivo JSON com o resultado da execução

dos testes

Listagem 17 - Código-fonte do teste onde a primeira falha exemplificada foi observada

Listagem 18 - Código-fonte do teste onde a segunda falha exemplificada foi observada 


\section{Lista de Abreviações}

BD Banco de Dados

BNF Backus-Naur Form

CTSVO Casos de Teste Semânticos Valorados e com Oráculo

DCTU Descrição Textual de Casos de Uso

FA Fluxo Alternativo

FC Fluxo Cancelador

FD Fluxo Disparador

FEI Fluxo de Exceção Irrecuperável

FER Fluxo de Exceção Recuperável

FP Fluxo Principal

FR Fluxo Retornável

FT Fluxo Terminador

GUI Graphical User Interface

JSON Java Script Object Notation

ME Máquina de Estados

QMTC Quantidade Mínima de casos de Teste semânticos gerados por Cenário

RN Regra de Negócio

SST Software sob Teste

TDD Test-Driven Development

UML Unified Modeling Language

XML eXtensible Markup Language 